\title{
Pyomyositis: a difficult diagnosis of an emerging disease in Italian immunocompetent children
}

\author{
PS Buonuomo*1, A De Cunto², L Lancella ${ }^{3}$, C Bracaglia ${ }^{1}$, P Salierno², \\ $S$ Colafati ${ }^{4}$, L Lepore ${ }^{2}$, E Cortis ${ }^{1}$ and AG Ugazio ${ }^{1}$
}

Address: ${ }^{1}$ Department of Pediatrics, Pediatric Rheumatology, Ospedale Pediatrico "Bambino Gesù" IRCCS, Rome, Italy, ${ }^{2}$ Clinica Pediatrica di Trieste, IRCCS Burlo Garofolo, Trieste, Italy, ${ }^{3}$ Department of Pediatrics, Pediatric Infectious Disease, Ospedale Pediatrico "Bambino Gesù" IRCCS, Rome, Italy and ${ }^{4}$ Department of Radiology, Ospedale Pediatrico "Bambino Gesù" IRCCS, Rome, Italy

* Corresponding author

from I5 ${ }^{\text {th }}$ Paediatric Rheumatology European Society (PreS) Congress

London, UK. 14-17 September 2008

Published: 15 September 2008

Pediatric Rheumatology 2008, 6(SuppI I):PI42 doi:I0.II86/I546-0096-6-SI-PI42

This abstract is available from: http://www.ped-rheum.com/content/6/SI/PI42

(c) 2008 Buonuomo et al; licensee BioMed Central Ltd.

Seven male and 1 female child with primary pyomyositis (mean age 11,4 years, range $3-1$ ), were diagnosed in 2 Italian Pediatric Hospitals since 2005. The most frequently reported symptoms were fever, increasing hip pain with functional impairment, and general malaise for several days prior to admission.

None had an underlying disease or a compromised immune system. Laboratory studies showed an elevated C-reactive protein and erythrocyte sedimentation rate while the CPK level was always within normal range.

$\mathrm{X}$ Rays and ultrasonography of the region involved were unremarkable in all patients, while MRI was diagnostic.

Primary pyomyositis (PM) is a subacute, deep bacterial infection of the large skeletal muscles (often gluteal), generally related to Staphylococcus aureus infection (50-90\%), Mycobacterium tuberculosis, Streptococcus pyogenes or anaerobic bacteria. It is frequent in tropical areas, while very few cases have been reported in Europe and the U.S where it is usually associated with immune deficiency (HIV, diabetes, tumors, liver or renal disease, or organ transplantation). Neglected cases may result in overwhelming sepsis, which might prove fatal, or in the formation of abscesses, requiring surgery. Because of the lack of specificity of laboratory testing, high clinical suspicion and early radiological evaluation are the key to diagnosis.
MRI is the most effective and non invasive instrument in helping to diagnose and define the anatomic extent of the infection and differentiate between the early stage of diffused muscle inflammation and the subsequent abscess formation.

We believe MRI should be performed as soon as this condition is suspected. 